\title{
LISTADO DE EVALUADORES/AS 2019
}

Agulló Díaz, Carmen (Universitat de València)

Álvarez Castrillón, Jose Antonio (Universidad de Oviedo)

Álvarez Gallego, Alejandro (Universidad Nacional de Colombia, Colombia)

Almeida, Mária (Agrupamento de Escolas de Casquilhos/UIED-FCT-UNL, Portugal)

Araújo de Oliveira, María Cristina (Universidade Federal de Juiz de Fora, Brasil)

Araujo, Marta (Universidad de Coimbra, Portugal)

Arnove, Robert (Indiana University, Estados Unidos)

Arredondo Marcos, Adelina (Universidad Autónoma del Estado de Morelos, México)

Ascenzi, Anna (Università di Macerata, Italia)

Ascolani, Adrián (Universidad Nacional de Rosario, Argentina)

Ayuso, María Luz (Universidad de Buenos Aires, Argentina)

Bencostta, Levy (Universidade Federal do Paraná, Brasil)

Cabrera Lafuente, Ana (Museo Nacional de Artes Decorativas)

Caldo, Paula (Universidad Nacional de Rosario, Argentina)

Canales Serrano, Antonio Fco. (Universidad Complutense de Madrid)

Carrillo Gallego, Dolores (Universidad de Murcia)

Carvalho, Marta Changas de (Universidade de São Paulo, Brasil)

Cid Castro, Eva (Universidad de Zaragoza)

Cordoví, Yoel (Instituto de Historia de Cuba, Cuba)

Costa, David Antonio da (Universidade Federal de Santa Catarina, Brasil)

Cruz Orozco, José I. (Universitat de València)

Davila Balsera, Pauli (Universidad del País Vasco)

Delgado Cendagortagalarza, Ander (Universidad del País Vasco)

Delgado Granados, Patricia (Universidad de Sevilla)

Diez Minguela, Alfonso (Universitat de València)

Doval, Delfina (Universidad Nacional de Entre Ríos, Argentina)

Egido Gálvez, Inmaculada (Universidad Complutense de Madrid) 
Escolano Benito, Agustín (Universidad de Valladolid)

Español González, Luis (Universidad de La Rioja)

Francesca Rubí Comas (Universitat de les Illes Balears)

Gallego Preciados, Ana Isabel (Universidad de Castilla- La Mancha)

García Salmerón, Pilar (Universidad Nacional de Educación a Distancia)

Garmendia Larrañaga, Joxe (Universidad del País Vasco)

Ginés Espín, José (Universidad de Murcia)

González Madrid, Damián (Universidad de Castilla - La Mancha)

González Redondo, Francisco (Universidad Complutense de Madrid)

Gvirtz, Silvina (Universidad Nacional de San Martín, Argentina)

Helfenberger, Marianne (Swiss University of Distance Learning, Suiza)

Herrera, Martha Cecilia (Universidad Pedagógica Nacional, Colombia)

Jover Olmeda, Gonzalo (Universidad Complutense de Madrid)

Kaufmann, Carolina (Universidad Nacional de Rosario, Argentina)

Mainer Braquer, Juan (Fedicaria)

Martín Zúñiga, Francisco (Universidad de Málaga)

Maurandi López, Antonio (Universidad de Murcia)

Mauri Medrano, Marta (Universidad de Zaragoza)

Maz Machado, Alexander (Universidad de Córdoba)

Meda, Juri (Università di Macerata, Italia)

Mendes, Iran Abreu (Universidade Federal do Rio Grande do Norte - Brasil)

Moreno Martínez, Pedro Luis (Universidad de Murcia)

Negrín Fajardo, Olegario (Universidad Nacional de Educación a Distancia)

Neto, Oscar Silva (Universidade Federal de Santa Catarina, Brasil)

Olivares, Pilar (Universidad de Murcia)

Ossenbach Sauter, Gabriela (Universidad Nacional de Educación a Distancia)

Pineau, Pablo (Universidad de Buenos Aires, Argentina)

Pintassilgo, Joaquim (Universidade de Lisboa, Portugal)

Pozo Andrés, María del Mar del (Universidad de Alcalá)

Rabazas, Teresa (Universidad Complutense de Madrid)

Rebollo, María José (Universidad de Sevilla)

Ríos Zúñiga, Rosalina (Universidad Nacional Autónoma de México, México)

Rodríguez Méndez, Francisco Javier (Universidad de Salamanca)

Sáenz Obregón, Javier (Universidad Nacional de Colombia, Colombia)

Sánchez Jiménez, Encarna (Universidad de Murcia)

Sánchez, Clara Helena (Universidad Nacional de Colombia)

Sánchez-Pedreño Guillén, Salvador ( Universidad de Murcia)

Simon, Frank (Universiteit Gent, Bélgica) 
Solà Gussinyer, Pere (Universitat Autònoma de Barcelona)

Somoza Rodríguez, Miguel (Universidad Nacional de Educación a Distancia)

Stam, Talitha (Erasmus University Rotterdam, Países Bajos)

Sureda García, Bernat (Universitat de les Illes Balears)

Terrón Bañuelos, Aida (Universidad de Oviedo)

Vea, Fernando (Universidad de Zaragoza)

Vicente Ferris, José Luis (Universidad Miguel Hernández)

Vilanou Torrano, Conrad (Universitat de Barcelona)

Villela Pereira, Marcos (Pontifícia Universidade Católica do Rio Grande do Sul, Brasil)

Viñao Frago, Antonio (Universidad de Murcia)

Welti, Elisa (Universidad Nacional de Rosario, Argentina) 